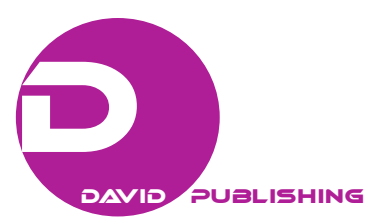

\title{
Development of Wages and Salaries of Czech Employees During the Last Period by Educational Attainment and Forecasts for 2011 and 2012
}

\author{
Diana Bílková \\ Department of Statistics and Probability, Faculty of Informatics and Statistics, University of Economics, Prague. \\ Department of Informatics and Mathematics, Faculty of Economic Studies, University of Finance and Administration, Prague
}

\begin{abstract}
This paper deals with the development of sample characteristics of wage distribution in recent years in the Czech Republic by the highest educational attainment. Gross monthly wage is the variable investigated. We distinguish the following scale of the highest educational attainment: primary and incomplete education, secondary education without GCSE, secondary education with GCSE, higher vocational and bachelor education and tertiary education. Forecasts of wage distribution have been developed for the next two years for all of these categories. Three-parametric lognormal curve formed the basis of the theoretical probability distribution. Parameter values of relevant three-parametric lognormal curves were then estimated using the method of L-moments of parameter estimation. Forecasts of sample values of L-moments were calculated using trend analysis of their past development and the parameters of three-parametric lognormal curves for forecasts of wage distribution were calculated using the predicted values of the first three sample L-moments. We have obtained the forecasts of wage distribution by the highest educational attainment on the basis of these probability density functions.
\end{abstract}

Keywords: Wage distribution, highest educational attainment, characteristics of location and differentiation, method of L-moment of parameter estimation, three-parametric lognormal distribution

\section{Introduction}

Major changes occurred in the Czech Republic during the period since the transition of the Czech economy from a centrally planned economy to a market economy. These changes related not only to the economy, but also to the areas, which are connected with economy. Wages and incomes of population increase and their differentiation increases markedly, too. The group of people with very high wages and incomes was created and it grows stronger gradually. The financial situation of Czech households changes, see for example Bartošová (2009), the purchasing power of Czech households changes, see Bartošová \& Forbelská (2010). Unemployment appeared and it develops gradually, see Miskolczi, Langhamrová \& Langhamrová (2011) or Löster \& Langhamrová (2011). Demographic structure of the population changes, see for example Fiala \&

Corresponding author: Diana Bílková, Department of Statistics and Probability, Faculty of Informatics and Statistics, University of Economics, Sq. W. Churchill 1938/4, 13067 Prague 3, E-mail: bilkova@vse.cz. Department of Informatics and Mathematics, Faculty of Economic Studies, University of Finance and Administration, Estonian Street 500/3, 10100 Prague 10, E-mail: diana.bilkova@vsfs.cz. 
Langhamrová (2011), Fiala \& Langhamrová (2010) or Malá (2011).

We can use various methods for processing data on wages and incomes, see for example Bartošová \& Bína, (2007) or Řezanková, Löster \& Hušek (2011). L-moments and L-moment method of parameter estimation are described in numerous professional publications and articles, for example Guttman (1993), Hosking (1990), Hosking \& Wales (1997) or Kyselý \& Picek (2007).

The studied variable for this research was a gross monthly wage (in CZK) and we came out of a set of employees classified by educational attainment in the categories: primary and incomplete education, secondary education without GCSE, secondary education with GCSE, higher vocational and bachelor education and tertiary education. The data come from the official website of the Czech Statistical Office. Table 1 provides information about the sample sizes of the individual sets used. The data were processed using the statistical programs SAS and Statgraphics and using the spreadsheet Microsoft Excel.

Table 1

Sample sizes of the wage distribution broken down by educational attainment

\begin{tabular}{|c|c|c|c|c|c|c|c|c|}
\hline \multirow{2}{*}{ Highest educational attain. } & \multicolumn{8}{|l|}{ Year } \\
\hline & 2003 & 2004 & 2005 & 2006 & 2007 & 2008 & 2009 & 2010 \\
\hline Primary and incomplete & 95,112 & 119,480 & 125,972 & 129,027 & 135,399 & 137,190 & 120,254 & 116,383 \\
\hline Secondary without GCSE & 377,347 & 470,688 & 523,744 & 553,522 & 587,081 & 591,669 & 557,780 & 555,266 \\
\hline Secondary with GCSE & 408,562 & 560,237 & 575,668 & 621,306 & 629,447 & 644,576 & 625,631 & 627,073 \\
\hline Higher vocational and bachelor & 15,749 & 29,144 & 40,055 & 42,856 & 47,967 & 54,439 & 57,747 & 64,684 \\
\hline Tertiary & 122,164 & 224,947 & 250,088 & 267,661 & 273,604 & 283,937 & 290,094 & 299,423 \\
\hline
\end{tabular}

Source: www.czso.cz

\section{Wage Distribution in the Czech Republic in 2003-2010}

Tables 2, 4, 6, 8 and 10 provide an overview of the development of sample characteristics of wage distribution in the Czech Republic in 2003-2010 by educational attainment. They are the characteristics of the location, such as arithmetic mean, median (middle value) and medial (half the sum); the characteristics of variability, such as standard deviation (characteristic of absolute variability) and coefficient of variation (characteristic of relative variability) and the characteristic of shape, such as moment measure of skewness and moment measure of kurtosis. We can see from these tables that the characteristics of the location increase in time, while the relation between the mentioned characteristics of the location is valid median $<$ arithmetic mean $<$ medial (see Figure 2 for the tertiary education), which is typical for the distribution with positively skewness. The development of the mentioned characteristics of location in 2003-2010 according to the highest educational attainment is also captured by Fig. 1. We can see from Tables 2, 4, 6, 8 and 10 and from Figs. 1 and 2 that wage growth has virtually stopped from 2008 onwards, therefore, apparently in connection with the onset of the global economic recession. We can also note fom these tables that the characteristic of absolute variability increases until 2008, with the exception of tertiary education. We can also see that all the researched wage distribution are characterized by positive skewness, which is typical for the wage and income distribution.

Tables 3, 5, 7, 9 and 11 present the first three sample L-moments for individual wage distribution, the parameter estimations calculated using the L-moment method of parameter estimation, the values of Gini's coefficient, and the sum $S$ of all absolute deviations between empirical and theoretical frequencies for all 
intervals, all for the years 2003-2010 according to the highest educational attainment. The trend functions for the period of 2003-2010 were constructed for the first three sample L-moments of the wage distribution. The values of the first three sample L-moments were predicted based on the development of their trend functions. Parameter values for three-parametric lognormal curves for the years 2011 and 2012 were calculated using the predicted values of the first three sample L-moments. Figs. 3-7 show the development of probability density functions of three-parametric lognormal curves of wage distributions in the years 2003-2010 by the highest educational attainment, including their forecasts for 2011 and 2012. Figs. 8-10 show a different shape of the probability density function by the highest educational attainment, both in the first year of the followed period and, in the last year of the followed period and for the second year of the forecasts.

Table 2

Sample characteristics of location, variability and of the shape of the wage distribution broken down by educational attainment - Primary and incomplete education

\begin{tabular}{lllllllll}
\hline & \multicolumn{1}{l}{ Year } \\
\cline { 2 - 8 } Characteristic & 2003 & 2004 & 2005 & 2006 & 2007 & 2008 & 2009 & 2010 \\
\hline $\begin{array}{l}\text { Arithmetic mean } \\
\text { (in CZK) }\end{array}$ & $12,504.190$ & $12,620.511$ & $13,286.119$ & $14,434.339$ & $14,863.200$ & $16,000.565$ & $15,663.997$ & $16,094.348$ \\
Median (in CZK) & $11,806.941$ & $12,538.417$ & $12,948.386$ & $13,598.293$ & $14,644.534$ & $15,682.416$ & $15,398.930$ & $15,753.463$ \\
Medial (in CZK) & $13,693.502$ & $14,819.126$ & $15,330.578$ & $16,124.914$ & $17,518.896$ & $18,537.361$ & $18,055.888$ & $18,490.163$ \\
$\begin{array}{l}\text { Standard deviation } \\
\text { in CZK) }\end{array}$ & $5,233.745$ & $6,422.459$ & $6,658.778$ & $7,215.889$ & $7,550.601$ & $7,899.442$ & $7,530.222$ & $7,580.522$ \\
Coefficient of & 41.86 & 50.89 & 50.12 & 49.99 & 50.80 & 49.37 & 48.07 & 47.10 \\
variation (in \%) & 0.800529 & 1.100331 & 1.139141 & 1.410259 & 0.952889 & 0.914085 & 0.824256 & 0.777434 \\
Skewness & 1.552571 & 3.295742 & 3.395737 & 4.254976 & 2.026614 & 1.926393 & 1.725168 & 1.574092 \\
Kurtosis & & & & & & & & \\
\hline
\end{tabular}

Source: Own computations

Table 3

Sample L-moments, parameter estimations of three-parametric lognormal curves obtained using the L-moment method, values of Gini's coefficient (past years and predictions for 2011 and 2012) and sum all absolute deviations of the observed and theoretical frequencies of all intervals broken down by educational attainmentPrimary and incomplete education

\begin{tabular}{|c|c|c|c|c|c|c|c|c|}
\hline \multirow[b]{2}{*}{ Year } & \multicolumn{3}{|c|}{ Sample L-moments } & \multicolumn{3}{|c|}{ Parameter estimations } & \multirow{2}{*}{\multicolumn{2}{|c|}{-Gini`s coefficient Criterion $S$}} \\
\hline & $l_{1}$ & $l_{2}$ & $l_{3}$ & $\mu$ & $\sigma^{2}$ & $\theta$ & & \\
\hline 2003 & $12,504.190$ & $2,811.582$ & 154.419 & 9.559814 & 0.112406 & $-2,498.99$ & 0.224851 & $15,719.98$ \\
\hline 2004 & $12,620.511$ & $3,369.199$ & 76.541 & 10.209928 & 0.046468 & $-15,189.78$ & 0.266962 & $24,066.43$ \\
\hline 2005 & $13,286.119$ & $3,485.083$ & 19.043 & 10.970985 & 0.011175 & $-45,201.64$ & 0.262310 & $58,062.62$ \\
\hline 2006 & $14,434.339$ & $3,694.085$ & 146.826 & 10.007644 & 0.081318 & $-8,682.19$ & 0.255923 & $31,022.25$ \\
\hline 2007 & $14,863.200$ & $4,030.671$ & 99.702 & 10.344902 & 0.050596 & $-17,031.66$ & 0.271185 & $29,510.78$ \\
\hline 2008 & $16,000.565$ & $4,227.564$ & 29.479 & 11.040909 & 0.014261 & $-46,820.10$ & 0.264213 & $28,911.51$ \\
\hline 2009 & $15,663.997$ & $4,047.552$ & 79.654 & 10.467757 & 0.040252 & $-20,214.13$ & 0.258398 & $25,587.20$ \\
\hline 2010 & $16,094.348$ & $4,086.836$ & 57.724 & 10.648015 & 0.028888 & $-26,627.20$ & 0.253930 & $419,677.41$ \\
\hline 2011 & $16,529.700$ & $3,975.600$ & 54.100 & 10.639483 & 0.027832 & $-25,806.55$ & 0.240513 & - \\
\hline 2012 & $16,762.500$ & $3,792.300$ & 53.500 & 10.573827 & 0.028854 & $-22,903.67$ & 0.226237 & - \\
\hline
\end{tabular}

Source: Own computations 
Development of Wages and Salaries of Czech Employees During the Last Period by Educational 27 Attainment and Forecasts for 2011 and 2012

Table 4

Sample characteristics of location, variability and of the shape of the wage distribution broken down by educational attainment - Secondary education without GCSE

\begin{tabular}{|c|c|c|c|c|c|c|c|c|}
\hline \multirow{2}{*}{ Characteristic } & \multicolumn{8}{|l|}{ Year } \\
\hline & 2003 & 2004 & 2005 & 2006 & 2007 & 2008 & 2009 & 2010 \\
\hline $\begin{array}{l}\text { Arithmetic mean } \\
\text { (in CZK) }\end{array}$ & $15,140.236$ & $15,809.510$ & $16,565.195$ & $17,585.703$ & $18,772.966$ & $20,095.488$ & $19,530.785$ & $19,922.694$ \\
\hline Median (in CZK) & $14,501.952$ & $15,432.768$ & $16,074.423$ & $16,847.303$ & $18,190.981$ & $19,394.852$ & $18,912.333$ & $19,289.294$ \\
\hline Medial (in CZK) & $16,378.697$ & $17,632.424$ & $18,337.833$ & $19,284.310$ & $20,970.860$ & $22,290.889$ & $21,642.011$ & $22,136.965$ \\
\hline $\begin{array}{l}\text { Standard deviation } \\
\text { (in } \mathrm{CZK} \text { ) }\end{array}$ & $5,677.100$ & $6,625.825$ & $6,880.465$ & $7,185.551$ & $8,064.500$ & $8,450.937$ & $8,160.661$ & $8,397.448$ \\
\hline $\begin{array}{l}\text { Coefficient of } \\
\text { variation (in \%) }\end{array}$ & 37.50 & 41.91 & 41.54 & 40.86 & 42.96 & 42.05 & 41.78 & 42.15 \\
\hline Skewness & 0.669926 & 0.720702 & 0.781426 & 0.889565 & 0.698529 & 0.660371 & 0.65293 & 0.619120 \\
\hline Kurtosis & 1.114192 & 2.087550 & 2.167180 & 2.246373 & 1.441347 & 1.170579 & 1.263066 & 1.071873 \\
\hline
\end{tabular}

Source: Own computations

Table 5

Sample L-moments, parameter estimations of three-parametric lognormal curves obtained using the L-moment method, values of Ginils coefficient (past years and predictions for 2011 and 2012) and sum all absolute deviations of the observed and theoretical frequencies of all intervals broken down by educational attainmentSecondary education without GCSE

\begin{tabular}{|c|c|c|c|c|c|c|c|c|}
\hline \multirow[b]{2}{*}{ Year } & \multicolumn{3}{|c|}{ Sample L-moments } & \multicolumn{3}{|c|}{ Parameter estimations } & \multirow{2}{*}{-Gini`s coefficient } & \multirow{2}{*}{ Criterion $S$} \\
\hline & $l_{1}$ & $l_{2}$ & $l_{3}$ & $\mu$ & $\sigma^{2}$ & $\theta$ & & \\
\hline 2003 & $15,140.236$ & $3,085.584$ & 270.618 & 9.390138 & 0.179698 & $2,045.23$ & 0.203800 & $38,481.27$ \\
\hline 2004 & $15,809.510$ & $3,558.515$ & 27.806 & 10.810980 & 0.015981 & $-34,150.10$ & 0.225087 & $75,170.08$ \\
\hline 2005 & $16,565.195$ & $3,688.098$ & 103.890 & 10.188145 & 0.057621 & $-10,798.03$ & 0.222641 & $219,404.84$ \\
\hline 2006 & $17,585.703$ & $3,837.157$ & 229.187 & 9.824680 & 0.122258 & $-2,063.83$ & 0.218198 & $56,376.06$ \\
\hline 2007 & $18,772.966$ & $4,355.660$ & 119.102 & 10.370078 & 0.055933 & $-14,022.48$ & 0.232018 & $94,623.90$ \\
\hline 2008 & $20,095.488$ & $4,582.992$ & 226.999 & 10.104760 & 0.101358 & $-5,635.19$ & 0.228061 & $75,971.66$ \\
\hline 2009 & $19,530.785$ & $4,420.274$ & 168.982 & 10.207885 & 0.078212 & $-8,666.77$ & 0.226323 & $73,619.35$ \\
\hline 2010 & $19,922.694$ & $4,566.315$ & 192.249 & 10.188798 & 0.086141 & $-7,851.67$ & 0.229202 & $68,358.56$ \\
\hline 2011 & $20,367.300$ & $4,591.400$ & 220.400 & 10.123584 & 0.098228 & $-5,811.31$ & 0.225430 & - \\
\hline 2012 & $20,473.100$ & $4,552.100$ & 243.300 & 10.056544 & 0.109384 & $-4,144.96$ & 0.222345 & - \\
\hline
\end{tabular}

Source: Own computations

Table 6

Sample characteristics of location, variability and of the shape of the wage distribution broken down by educational attainment - Secondary education with GCSE

\begin{tabular}{|c|c|c|c|c|c|c|c|c|}
\hline \multirow[b]{2}{*}{ Characteristic } & \multicolumn{8}{|l|}{ Year } \\
\hline & 2003 & 2004 & 2005 & 2006 & 2007 & 2008 & 2009 & 2010 \\
\hline $\begin{array}{l}\text { Arithmetic mean } \\
\text { (in CZK) }\end{array}$ & $19,180.093$ & $20,752.937$ & $21,689.240$ & $22,806.853$ & $24,173.876$ & $25,641.295$ & $25,686.897$ & $25,846.068$ \\
\hline Median (in CZK) & $17,881.643$ & $19,212.329$ & $20,009.005$ & $21,058.124$ & $22,392.406$ & $23,680.970$ & $23,797.152$ & $24,027.505$ \\
\hline Medial (in CZK) & $20,118.182$ & $22,021.612$ & $22,993.469$ & $24,025.138$ & $25,853.715$ & $27,343.865$ & $27,369.635$ & $27,639.066$ \\
\hline $\begin{array}{l}\text { Standard deviation } \\
\text { (in } \mathrm{CZK} \text { ) }\end{array}$ & $6,961.509$ & $8,696.317$ & $8,923.621$ & $9,351.419$ & $9,869.296$ & $10,106.053$ & $10,090.622$ & $10,178.031$ \\
\hline $\begin{array}{l}\text { Coefficient of } \\
\text { variation (in \%) }\end{array}$ & 36.30 & 41.90 & 41.14 & 41.00 & 40.83 & 39.41 & 39.28 & 39.38 \\
\hline Skewness & 0.722822 & 1.193881 & 1.069475 & 1.008185 & 0.793520 & 0.684125 & 0.668208 & 0.616414 \\
\hline Kurtosis & 0.204638 & 2.045810 & 1.532053 & 1.066599 & 0.538724 & 0.162828 & 0.165829 & 0.117399 \\
\hline
\end{tabular}

Source: Own computations 
Table 7

Sample L-moments, parameter estimations of three-parametric lognormal curves obtained using the L-moment method, values of Ginils coefficient (past years and predictions for 2011 and 2012) and sum all absolute deviations of the observed and theoretical frequencies of all intervals broken down by educational attainmentSecondary education with GCSE

\begin{tabular}{lllllllll}
\hline \multirow{2}{*}{ Year } & \multicolumn{7}{l}{ Sample L-moments } & \multicolumn{7}{l}{ Parameter estimations } \\
\cline { 2 - 9 } & $l_{1}$ & $l_{2}$ & $l_{3}$ & $\mu$ & $\sigma^{2}$ & $\theta$ & Gini`s coefficient Criterion $S$ \\
\hline 2003 & $19,180.093$ & $3,798.195$ & 614.977 & 9.224972 & 0.333221 & $7,193.04$ & 0.198028 & $53,028.37$ \\
2004 & $20,752.937$ & $4,546.402$ & 724.770 & 9.414827 & 0.328019 & $6,297.35$ & 0.219073 & $87,974.32$ \\
2005 & $21,689.240$ & $4,719.647$ & 754.682 & 9.450260 & 0.329031 & $6,704.68$ & 0.217603 & $205,065.51$ \\
2006 & $22,806.853$ & $4,980.515$ & 852.397 & 9.459815 & 0.352485 & $7,499.98$ & 0.218378 & $83,978.83$ \\
2007 & $24,173.876$ & $5,334.162$ & 825.794 & 9.593459 & 0.318435 & $6,973.67$ & 0.220658 & $84,596.17$ \\
2008 & $25,641.295$ & $5,515.480$ & 895.508 & 9.596208 & 0.334158 & $8,257.61$ & 0.215101 & $81,751.93$ \\
2009 & $25,686.897$ & $5,509.906$ & 875.497 & 9.609149 & 0.326934 & $8,140.29$ & 0.214503 & $84,955.38$ \\
2010 & $25,846.068$ & $5,570.312$ & 844.566 & 9.650096 & 0.311793 & $7,703.92$ & 0.215519 & $89,232.71$ \\
2011 & $26,388.300$ & $5,473.200$ & 810.100 & 9.647814 & 0.304296 & $8,355.24$ & 0.207410 & - \\
2012 & $26,485.800$ & $5,315.400$ & 752.200 & 9.646879 & 0.290804 & $8,590.72$ & 0.200689 & - \\
\hline
\end{tabular}

Source: Own computations

Table 8

Sample characteristics of location, variability and of the shape of the wage distribution broken down by educational attainment - Higher vocational and bachelor education

\begin{tabular}{lllllllll}
\hline & \multicolumn{7}{l}{ Year } \\
\cline { 2 - 8 } Characteristic & 2003 & 2004 & 2005 & 2006 & 2007 & 2008 & 2009 & 2010 \\
\hline $\begin{array}{l}\text { Arithmetic mean } \\
\text { (in CZK) }\end{array}$ & $20,398.622$ & $22,871.093$ & $24,253.633$ & $25,337.526$ & $26,810.390$ & $28,533.110$ & $29,033.179$ & $29,220.425$ \\
Median (in CZK) & $18,955.765$ & $20,750.186$ & $22,164.165$ & $22,874.456$ & $24,647.127$ & $26,281.795$ & $26,802.167$ & $26,984.813$ \\
Medial (in CZK) & $21,348.335$ & $24,267.180$ & $25,899.683$ & $26,571.459$ & $28,699.964$ & $30,592.367$ & $31,096.222$ & $31,289.758$ \\
$\begin{array}{l}\text { Standard deviation } \\
\text { (in CZK) }\end{array}$ & $7,143.562$ & $9,723.674$ & $9,944.143$ & $10,123.983$ & $10,368.726$ & $10,675.919$ & $10,639.565$ & $10,607.755$ \\
Coefficient of & 35.02 & 42.52 & 41.00 & 39.96 & 38.67 & 37.42 & 36.65 & 36.30 \\
variation (in \%) & 0.648034 & 1.046529 & 0.898087 & 0.910353 & 0.667752 & 0.527284 & 0.481307 & 0.472111 \\
Skewness & -0.123202 & 0.923240 & 0.418578 & 0.218218 & -0.207604 & -0.545582 & -0.594228 & -0.616237 \\
Kurtosis & & & & & & & & \\
\hline
\end{tabular}

Source: Own computations

Table 9

Sample L-moments, parameter estimations of three-parametric lognormal curves obtained using the L-moment method, values of Ginils coefficient (past years and predictions for 2011 and 2012) and sum all absolute deviations of the observed and theoretical frequencies of all intervals broken down by educational attainmentHigher vocational and bachelor education

\begin{tabular}{lllllllll}
\hline \multirow{2}{*}{ Year } & \multicolumn{9}{l}{ Sample L-moments } & \multicolumn{7}{l}{ Parameter estimations } & \multicolumn{2}{c}{ Gini`s coefficient Criterion $S$} \\
\cline { 2 - 8 } & $l_{1}$ & $l_{2}$ & $l_{3}$ & $\mu$ & $\sigma^{2}$ & $\theta$ & \\
2003 & $20,398.622$ & $3,913.242$ & 666.593 & 9.221736 & 0.350807 & $8,344.77$ & 0.191839 & $2,593.48$ \\
2004 & $22,871.093$ & $5,173.634$ & 982.521 & 9.428570 & 0.391772 & $7,740.76$ & 0.226208 & $3,480.52$ \\
2005 & $24,253.633$ & $5,371.772$ & 985.065 & 9.489684 & 0.378075 & $8,279.57$ & 0.221483 & $11,053.98$ \\
\hline
\end{tabular}


Development of Wages and Salaries of Czech Employees During the Last Period by Educational 29 29 Attainment and Forecasts for 2011 and 2012

Table 9 continued

\begin{tabular}{cllllllll}
\hline \multirow{2}{*}{ Year } & \multicolumn{7}{l}{ Sample L-moments } & \multicolumn{7}{l}{ Parameter estimations } \\
\cline { 2 - 9 } & $l_{1}$ & $l_{2}$ & $l_{3}$ & $\mu$ & $\sigma^{2}$ & $\theta$ & Gini`s coefficient Criterion $S$ \\
\hline 2006 & $25,337.526$ & $5,452.000$ & $1,113.388$ & 9.431366 & 0.421868 & $9,934,81$ & 0.215175 & $4,624.00$ \\
2007 & $26,810.390$ & $5,705.028$ & $1,032.978$ & 9.558354 & 0.373228 & $9,742.26$ & 0.212792 & $5,293.34$ \\
2008 & $28,533.110$ & $5,920.966$ & $1,084.820$ & 9.587615 & 0.377737 & $10,918.52$ & 0.207512 & $7,118.89$ \\
2009 & $29,033.179$ & $5,912.212$ & $1,059.253$ & 9.601053 & 0.369249 & $11,255.89$ & 0.203636 & $8,271.16$ \\
2010 & $29,220.425$ & $5,898.289$ & $1,057.590$ & 9.598172 & 0.369544 & $11,491.66$ & 0.201855 & $9,347.06$ \\
2011 & $29,759.200$ & $5,979.400$ & $1,115.200$ & 9.585500 & 0.384634 & $12,121.10$ & 0.200926 & - \\
2012 & $29,849.300$ & $6,010.400$ & $1,121.800$ & 9.590180 & 0.384919 & $12,125.92$ & 0.201358 & - \\
\hline
\end{tabular}

Source: Own computations

Table 10

Sample characteristics of location, variability and of the shape of the wage distribution broken down by educational attainment - Tertiary education

\begin{tabular}{lllllllll}
\hline & \multicolumn{1}{l}{ Year } \\
\cline { 2 - 8 } Characteristic & 2003 & 2004 & 2005 & 2006 & 2007 & 2008 & 2009 & 2010 \\
\hline $\begin{array}{l}\text { Arithmetic mean } \\
\text { (in CZK) }\end{array}$ & $26,337.703$ & $30,083.859$ & $31,363.739$ & $32,684.234$ & $34,293.881$ & $35,589.324$ & $36,013.778$ & $35,917.961$ \\
Median (in CZK) & $25,306.136$ & $27,003.114$ & $28,487.170$ & $30,136.182$ & $32,320.569$ & $34,643.011$ & $35,219.512$ & $35,169.460$ \\
Medial (in CZK) & $29,822.866$ & $33,081.061$ & $34,696.188$ & $36,398.605$ & $38,584.976$ & $40,372.060$ & $40,624.095$ & $40,784.054$ \\
$\begin{array}{l}\text { Standard deviation } \\
\text { (in CZK) }\end{array}$ & $7,558.736$ & $11,385.261$ & $11,283.875$ & $11,174.903$ & $11,077.456$ & $10,982.113$ & $10,822.634$ & $10,953.276$ \\
$\begin{array}{l}\text { Coefficient of } \\
\text { variation (in \%) }\end{array}$ & 28.70 & 37.85 & 35.98 & 34.19 & 32.30 & 30.86 & 30.05 & 30.50 \\
$\begin{array}{l}\text { Skewness } \\
\text { Kurtosis }\end{array}$ & 0.126549 & 0.432745 & 0.315120 & 0.203251 & 0.000530 & 0.174288 & 0.213793 & 0.205906 \\
\hline
\end{tabular}

Source: Own computations

Table 11

Sample L-moments, parameter estimations of three-parametric lognormal curves obtained using the L-moment method, values of Ginils coefficient (past years and predictions for 2011 and 2012) and sum all absolute deviations of the observed and theoretical frequencies of all intervals broken down by educational attainmentTertiary education

\begin{tabular}{lllllllll}
\hline \multirow{2}{*}{ Year } & \multicolumn{2}{l}{ Sample L-moments } & \multicolumn{7}{l}{ Parameter estimations } \\
\cline { 2 - 9 } & $l_{1}$ & $l_{2}$ & $l_{3}$ & $\mu$ & $\sigma^{2}$ & $\theta$ & Gini s coefficient Criterion $S$ \\
\hline 2003 & $26,337.703$ & $4,196.665$ & 548.566 & 9.459961 & 0.268424 & $11,658.70$ & 0.159341 & $46,971.92$ \\
2004 & $30,083.859$ & $6,330.912$ & $1,215.556$ & 9.623017 & 0.396169 & $11,665.52$ & 0.210442 & $45,273.10$ \\
2005 & $31,363.739$ & $6,301.118$ & $1,115.360$ & 9.672787 & 0.364742 & $12,307.35$ & 0.200905 & $92,541.14$ \\
2006 & $32,684.234$ & $6,248.776$ & $1,057.879$ & 9.693804 & 0.348617 & $13,379.37$ & 0.191186 & $69,656.17$ \\
2007 & $34,293.881$ & $6,187.184$ & $1,011.116$ & 9.706916 & 0.336364 & $14,853.68$ & 0.180417 & $88,553.90$ \\
2008 & $35,589.324$ & $6,091.293$ & 882.144 & 9.768596 & 0.297668 & $15,308.53$ & 0.171155 & $104,677.64$ \\
2009 & $36,013.778$ & $5,987.473$ & 829.063 & 9.779570 & 0.284484 & $15,643.92$ & 0.166255 & $107,226.99$ \\
2010 & $35,917.961$ & $6,061.156$ & 869.237 & 9.769798 & 0.294742 & $15,642.44$ & 0.168750 & $117,058.58$ \\
2011 & $35,708.400$ & $6,440.300$ & 527.800 & 10.164938 & 0.167875 & $7,457.65$ & 0.180358 & - \\
2012 & $35,014.200$ & $6,468.800$ & 262.700 & 10.556402 & 0.083088 & $-5,038.27$ & 0.184748 & - \\
\hline
\end{tabular}

Source: Own computations 


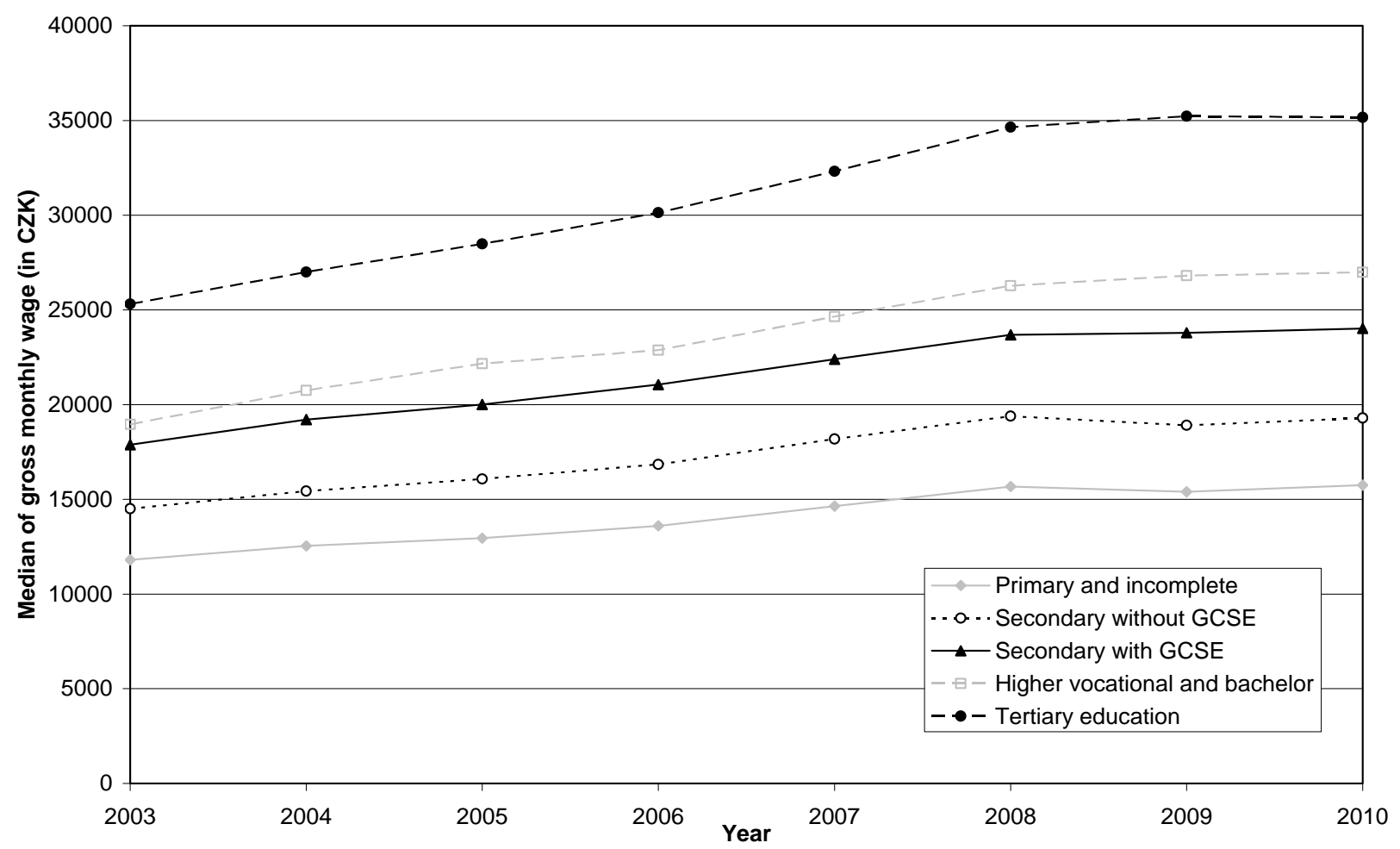

Fig. 1. Development of median of gross monthly wage (in CZK) in the years 2003-2010 by the highest educational attainment, Source: Own computations.

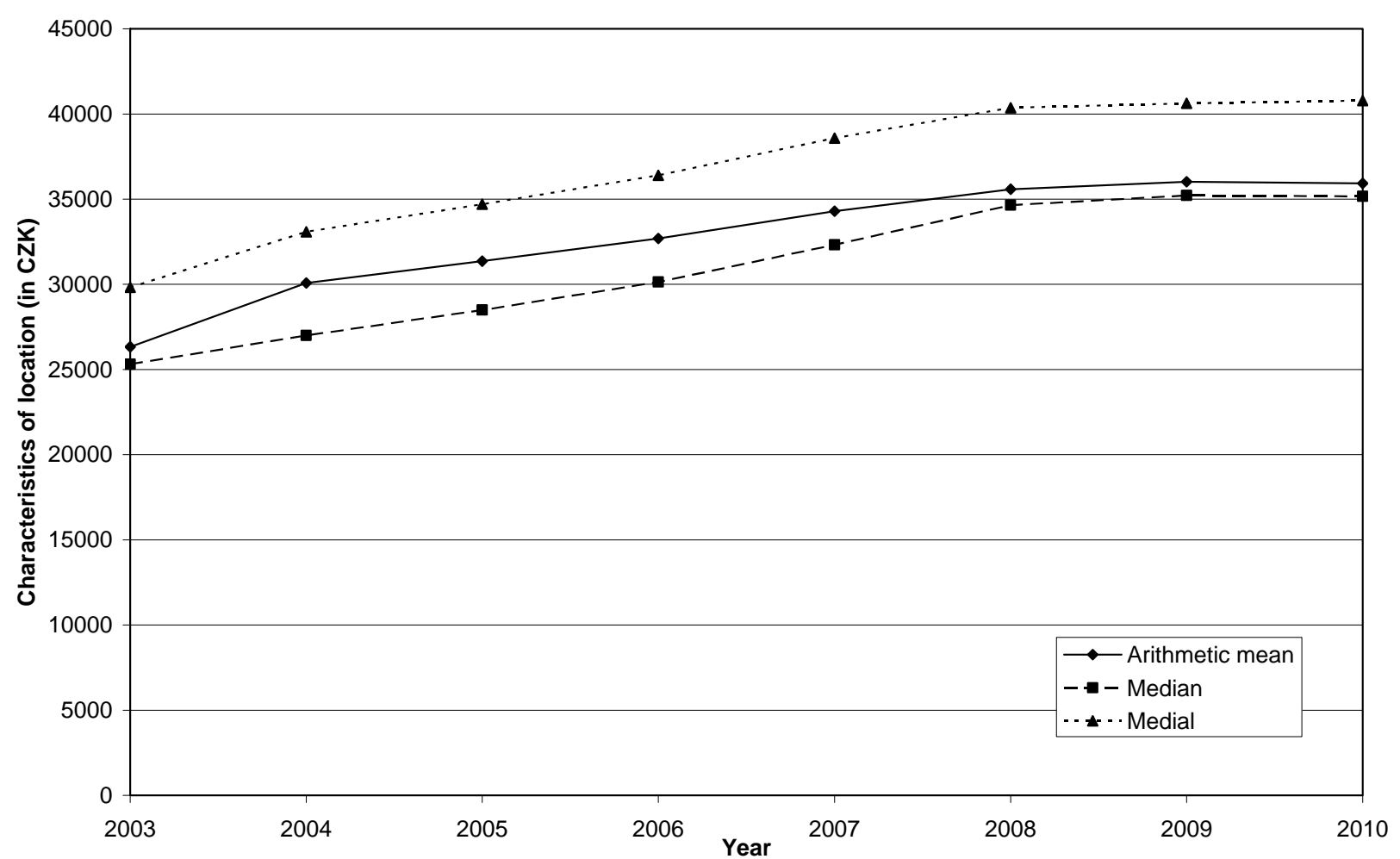

Figure 2. Development of characteristics of location of gross monthly wage (in CZK) in the years 2003-2010 for tertiary education, Source: Own computations 
Development of Wages and Salaries of Czech Employees During the Last Period by Educational 3 Attainment and Forecasts for 2011 and 2012

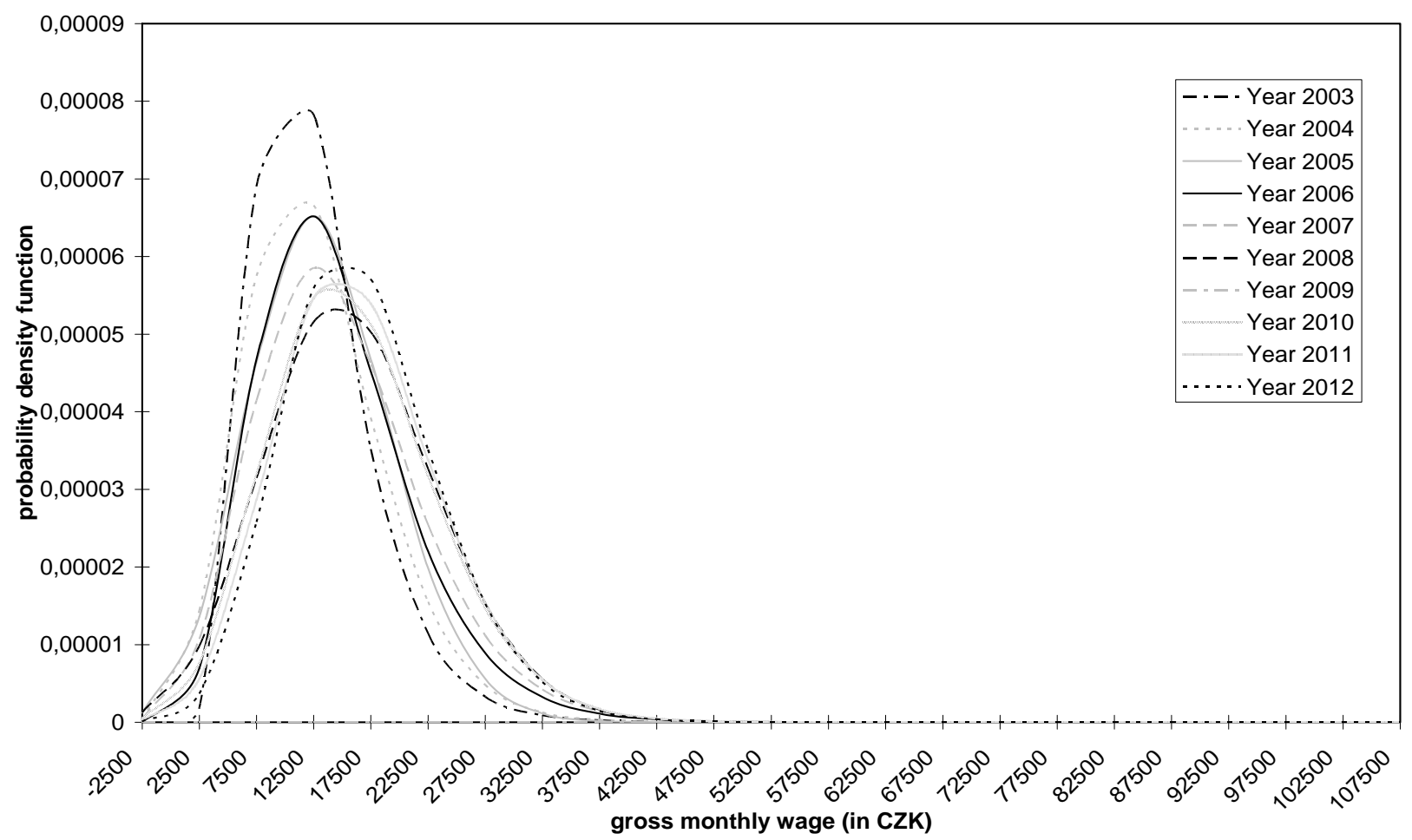

Figure 3. Probability density function including the predictions by the highest educational attainment - Primary and incomplete education, Source: Own computations.

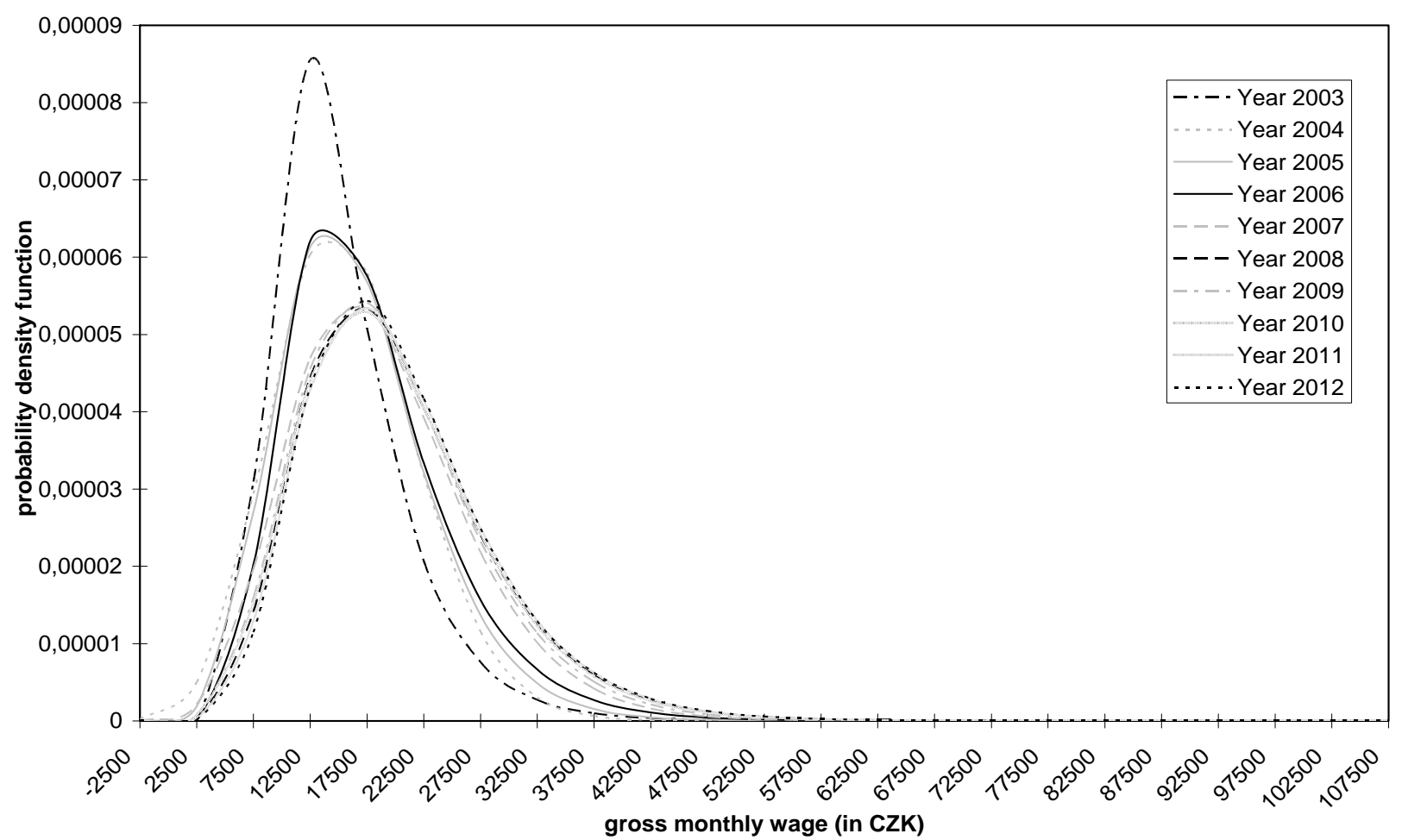

Figure 4. Probability density function including the predictions by the highest educational attainment - Secondary education without GCSE, Source: Own computations 


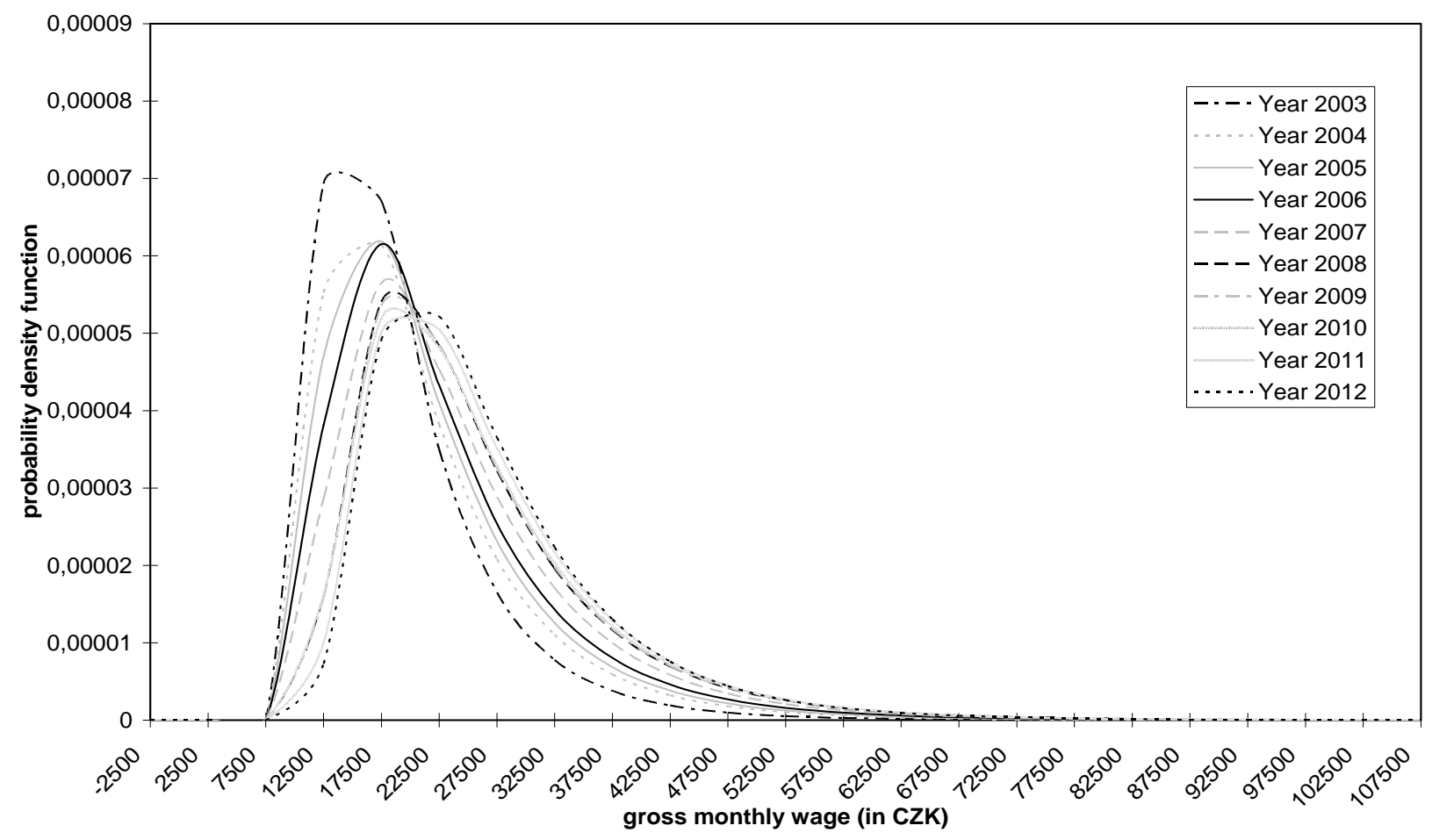

Figure 5. Probability density function including the predictions by the highest educational attainment - Secondary education with GCSE, Source: Own computations

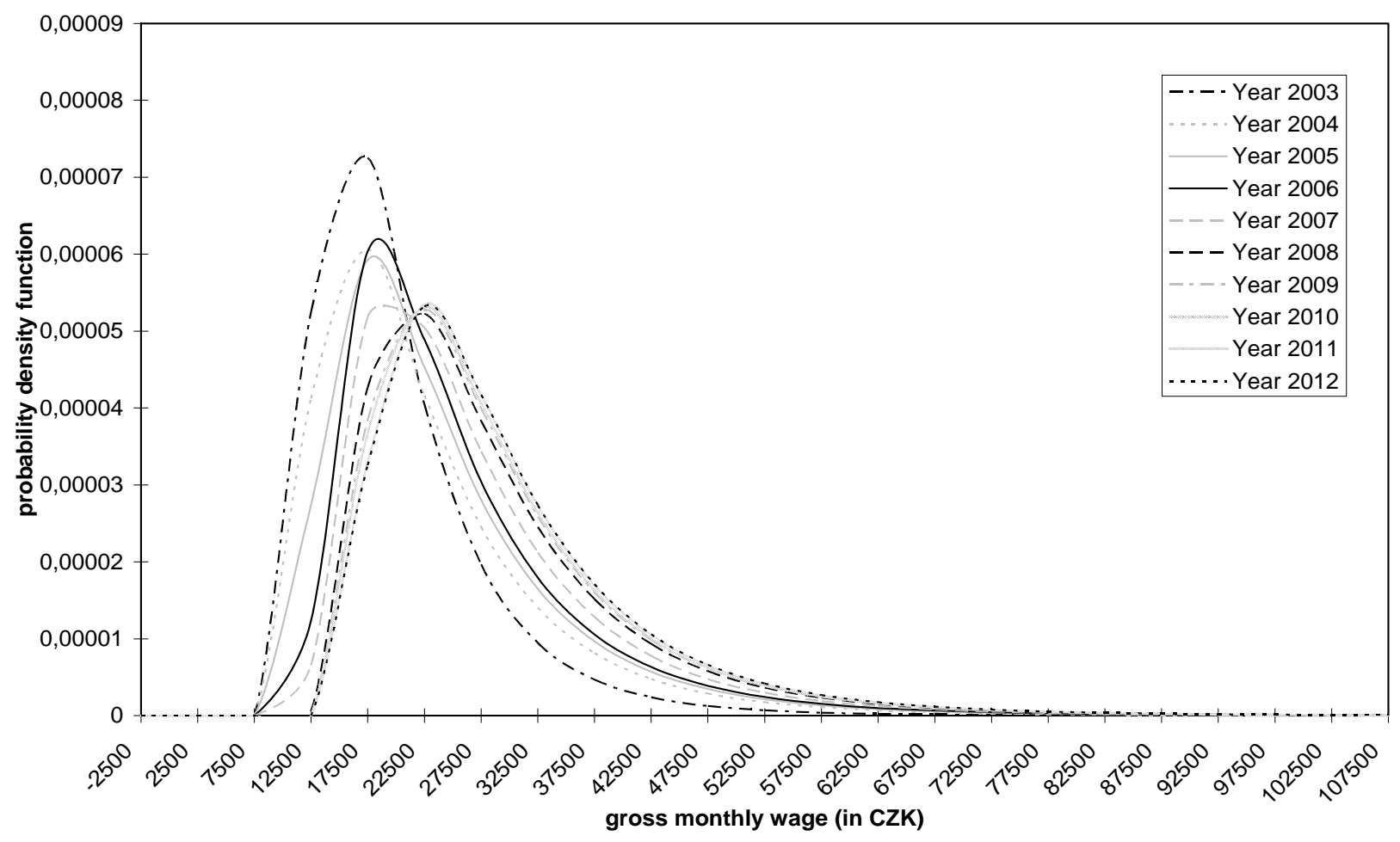

Figure 6. Probability density function including the predictions by the highest educational attainment - Higher vocational and bachelor education, Source: Own computations 
Development of Wages and Salaries of Czech Employees During the Last Period by Educational 33 Attainment and Forecasts for 2011 and 2012

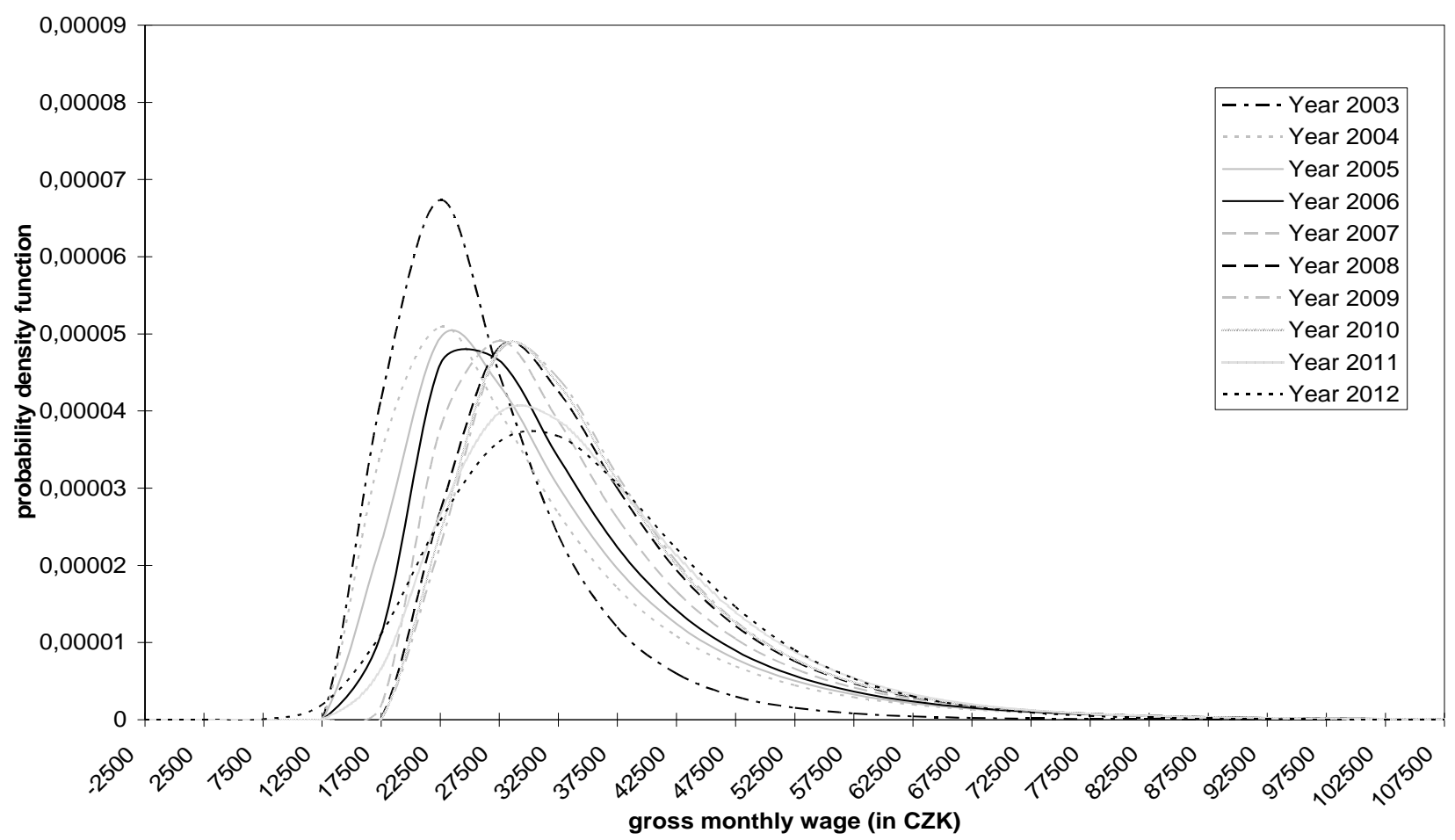

Figure 7. Probability density function including the predictions by the highest educational attainment - Tertiary education, Source: Own computations

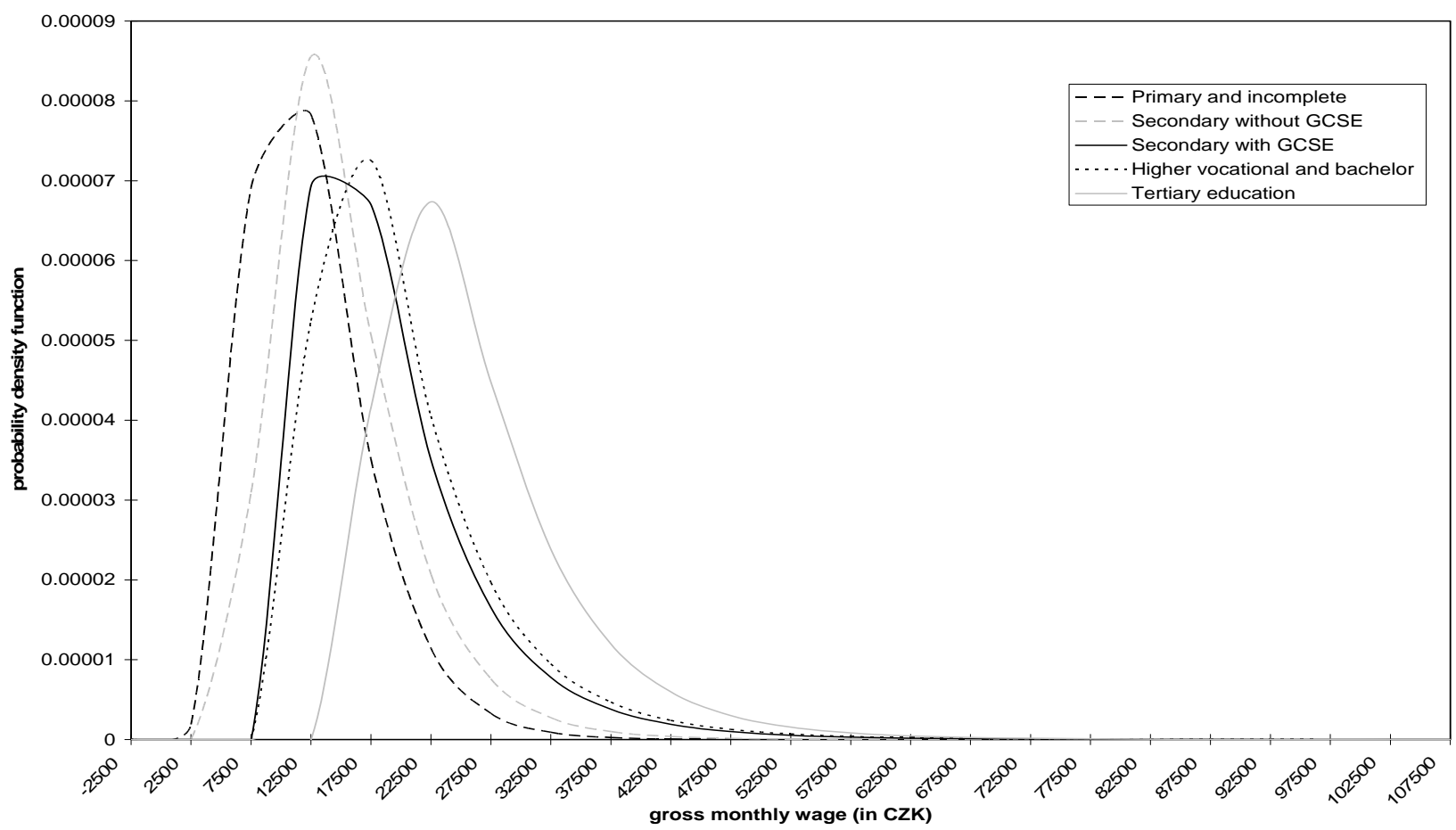

Figure 8. Probability density function by the highest educational attainment in 2003, Source: Own computations. 


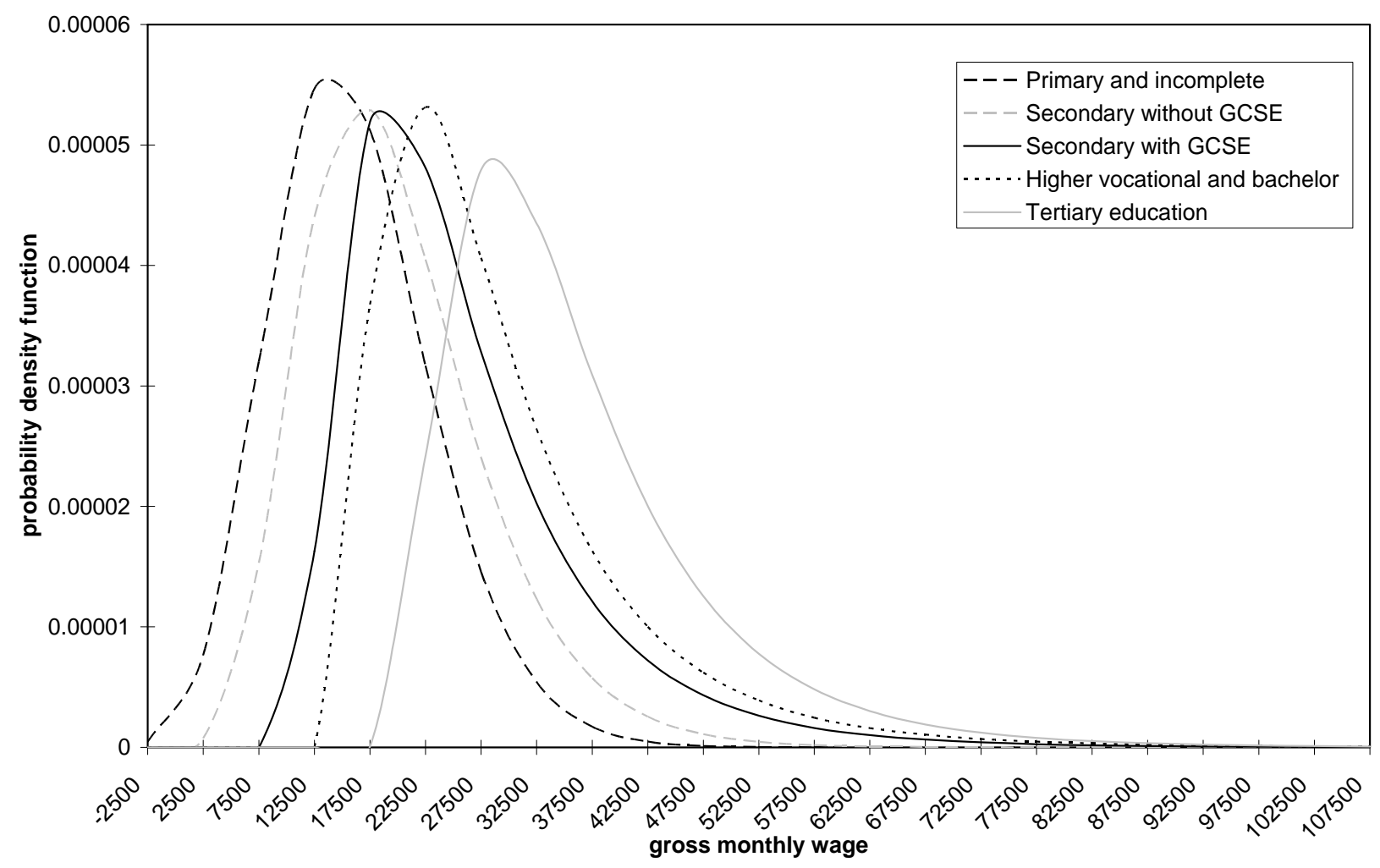

Figure 9. Probability density function by the highest educational attainment in 2010 , Source: Own computations

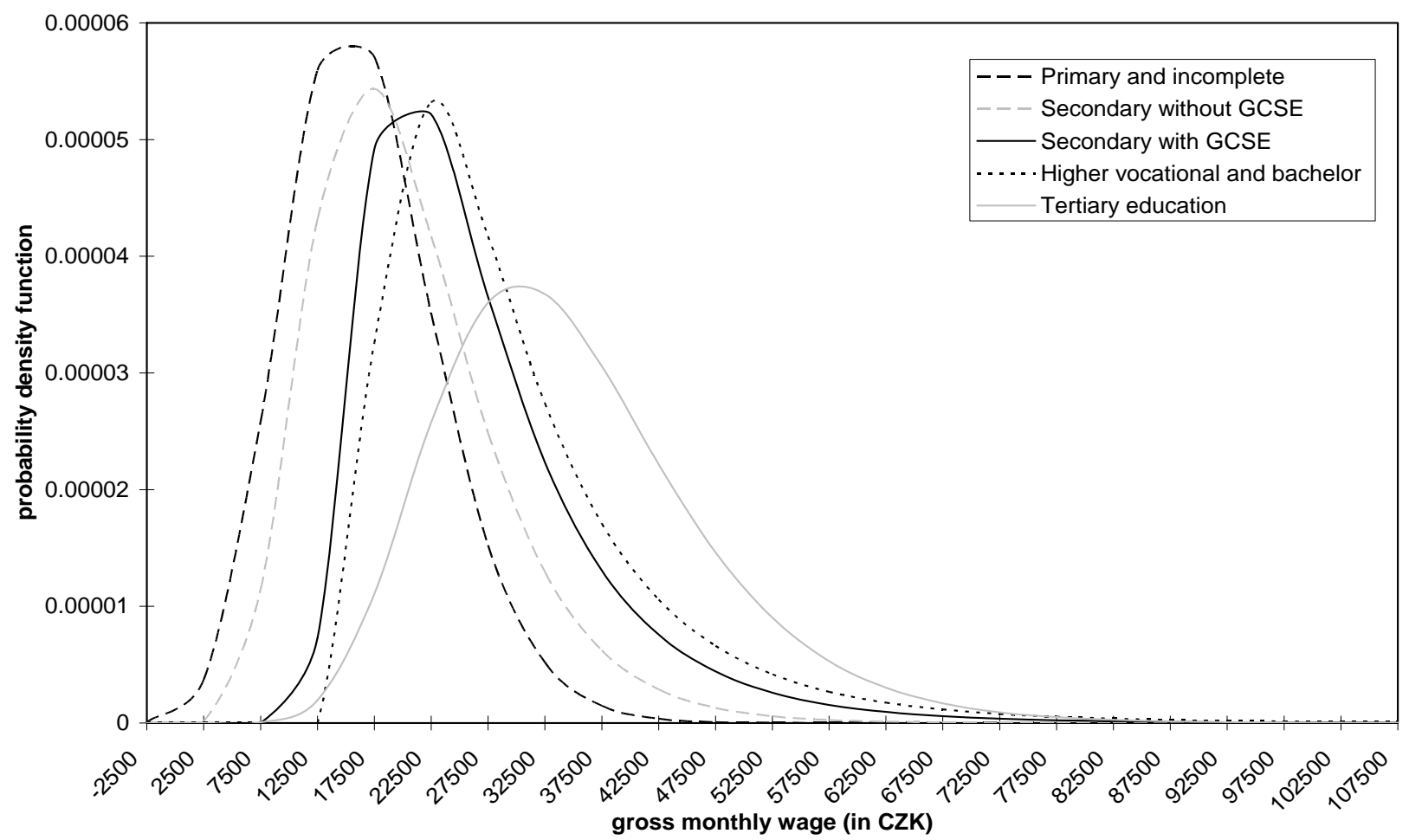

Figure 10. Probability density function by the highest educational attainment - predictions for 2012, Source: Own computations 
Development of Wages and Salaries of Czech Employees During the Last Period by Educational 35 Attainment and Forecasts for 2011 and 2012

\section{Conclusion}

Forecasts of the wage distribution by the highest educational attainment were calculated based on the model three-parametric lognormal curves for the years 2011 and 2012, see Table 12.

Strong difference in the level of gross monthly wage is evident by the highest educational attainment. The level of wage increases markedly with the highest educational attainment.

Table 12

Predictions of wage distributions for 2011 and 2012 according to highest level of education

\begin{tabular}{|c|c|c|c|c|c|c|c|c|c|c|c|c|}
\hline \multirow[b]{3}{*}{ Interval } & & \multirow[b]{3}{*}{ Year } & \multicolumn{10}{|c|}{ Highest level of education } \\
\hline & & & \multicolumn{2}{|c|}{$\begin{array}{l}\text { Primary and } \\
\text { incomplete } \\
\text { education }\end{array}$} & \multicolumn{2}{|c|}{$\begin{array}{l}\text { Secondary } \\
\text { education } \\
\text { without GCSE }\end{array}$} & \multicolumn{2}{|c|}{$\begin{array}{l}\text { Secondary } \\
\text { education } \\
\text { with GCSE }\end{array}$} & \multicolumn{2}{|c|}{$\begin{array}{l}\text { Higher } \\
\text { vocational and } \\
\text { bachelor education }\end{array}$} & \multicolumn{2}{|c|}{$\begin{array}{l}\text { Tertiary } \\
\text { education }\end{array}$} \\
\hline & & & 2011 & 2012 & 2011 & 2012 & 2011 & 2012 & 2011 & 2012 & 2011 & 2012 \\
\hline 0 & - & 5,000 & 3.42 & 2.35 & 0.38 & 0.23 & 0.00 & 0.00 & 0.00 & 0.00 & 0.00 & 0.00 \\
\hline 5,001 & - & 10,000 & 14.44 & 13.14 & 6.94 & 6.32 & 0.00 & 0.00 & 0.00 & 0.00 & 0.00 & 0.06 \\
\hline 10,001 & - & 15,000 & 26.68 & 27.26 & 20.93 & 21.04 & 6.25 & 5.11 & 0.45 & 0.44 & 0.13 & 1.14 \\
\hline 15,001 & - & 20,000 & 26.53 & 28.02 & 26.19 & 26.65 & 24.01 & 23.49 & 15.68 & 15.49 & 3.65 & 5.67 \\
\hline 20,001 & - & 25,000 & 16.96 & 17.64 & 20.62 & 20.79 & 24.93 & 25.73 & 26.07 & 25.95 & 13.12 & 12.79 \\
\hline 25,001 & - & 30,000 & 7.87 & 7.84 & 12.56 & 1.54 & 17.60 & 18.31 & 20.81 & 20.82 & 19.56 & 17.80 \\
\hline 30,001 & - & 35,000 & 2.89 & 2.71 & 6.59 & 6.57 & 10.93 & 11.28 & 13.71 & 13.77 & 19.22 & 18.23 \\
\hline 35,001 & - & 40,000 & 0.89 & 0.78 & 3.18 & 3.17 & 6.51 & 6.61 & 8.56 & 8.62 & 15.20 & 15.24 \\
\hline 40,001 & - & 45,000 & 0.24 & 0.20 & 1.45 & 1.47 & 3.84 & 3.83 & 5.29 & 5.34 & 10.68 & 11.10 \\
\hline 45,001 & - & 50,000 & 0.06 & 0.05 & 0.65 & 0.66 & 2.28 & 2.23 & 3.29 & 3.33 & 7.01 & 7.35 \\
\hline 50,001 & - & 55,000 & 0.02 & 0.01 & 0.28 & 0.30 & 1.37 & 1.31 & 2.08 & 2.10 & 4.42 & 4.55 \\
\hline 55,001 & - & 60,000 & 0.00 & 0.00 & 0.12 & 0.13 & 0.83 & 0.78 & 1.33 & 1.35 & 2.73 & 2.68 \\
\hline 60,001 & - & 65,000 & 0.00 & 0.00 & 0.05 & 0.06 & 0.51 & 0.48 & 0.87 & 0.88 & 1.67 & 1.53 \\
\hline 65,001 & - & 70,000 & 0.00 & 0.00 & 0.04 & 0.04 & 0.32 & 0.29 & 0.57 & 0.58 & 1.01 & 0.85 \\
\hline 70,001 & - & 75,000 & 0.00 & 0.00 & 0.02 & 0.02 & 0.21 & 0.18 & 0.39 & 0.39 & 0.62 & 0.47 \\
\hline 75,001 & - & 80,000 & 0.00 & 0.00 & 0.00 & 0.01 & 0.13 & 0.12 & 0.26 & 0.27 & 0.37 & 0.25 \\
\hline 80,001 & - & 85,000 & 0.00 & 0.00 & 0.00 & 0.00 & 0.09 & 0.08 & 0.18 & 0.19 & 0.23 & 0.14 \\
\hline 85,001 & - & 90,000 & 0.00 & 0.00 & 0.00 & 0.00 & 0.06 & 0.05 & 0.13 & 0.13 & 0.14 & 0.07 \\
\hline 90,001 & - & 95,000 & 0.00 & 0.00 & 0.00 & 0.00 & 0.04 & 0.04 & 0.09 & 0.09 & 0.09 & 0.04 \\
\hline 95,001 & - & 100,000 & 0.00 & 0.00 & 0.00 & 0.00 & 0.03 & 0.03 & 0.06 & 0.07 & 0.05 & 0.02 \\
\hline 100,001 & - & 105,000 & 0.00 & 0.00 & 0.00 & 0.00 & 0.02 & 0.02 & 0.05 & 0.05 & 0.04 & 0.01 \\
\hline 105,001 & - & 110,000 & 0.00 & 0.00 & 0.00 & 0.00 & 0.02 & 0.02 & 0.03 & 0.04 & 0.03 & 0.01 \\
\hline 110,001 & - & 115,000 & 0.00 & 0.00 & 0.00 & 0.00 & 0.01 & 0.01 & 0.03 & 0.03 & 0.01 & 0.00 \\
\hline 115,001 & - & 120,000 & 0.00 & 0.00 & 0.00 & 0.00 & 0.01 & 0.00 & 0.02 & 0.02 & 0.01 & 0.00 \\
\hline 120,001 & - & 125,000 & 0.00 & 0.00 & 0.00 & 0.00 & 0.00 & 0.00 & 0.02 & 0.02 & 0.01 & 0.00 \\
\hline 125,001 & - & 130,000 & 0.00 & 0.00 & 0.00 & 0.00 & 0.00 & 0.00 & 0.02 & 0.02 & 0.00 & 0.00 \\
\hline 130,001 & - & 135,000 & 0.00 & 0.00 & 0.00 & 0.00 & 0.00 & 0.00 & 0.01 & 0.01 & 0.00 & 0.00 \\
\hline 135,001 & - & $\infty$ & 0.00 & 0.00 & 0.00 & 0.00 & 0.00 & 0.00 & 0.00 & 0.00 & 0.00 & 0.00 \\
\hline \multicolumn{3}{|c|}{ Total (in \%) } & 100 & 100 & 100 & 100 & 100 & 100 & 100 & 100 & 100 & 100 \\
\hline
\end{tabular}

Source: Own computationAs expected, the graduates achieve the highest level of wage. For example, in the year 2003 the middle gross monthly wage of the university graduates was 1.42 times higher than for graduates of secondary vocational schools and 2.14 times higher than for workers with primary education. In the year 2010 the median of gross monthly wage of the university graduates was 1.46 times higher than for graduates of secondary vocational schools and 2.23 higher than for workers with primary education. 


\section{References}

Bartošová, J. (2009). Analysis and Modelling of Financial Power of Czech Households. Bratislava 03.02.2009 - 06.02.2009. In: $8^{\text {th }}$ International Conference APLIMAT (Part I). Bratislava: Slovak University of Technology in Bratislava, pp. 717-722.

Bartošová, J. \& Bína, V. (2007). Mixture Models of Household Income Distribution in the Czech Republic. Bratislava 06.02.2007 - 09.02.2007. In: $6^{\text {th }}$ International Conference APLIMAT (Part I). Bratislava: Slovak University of Technology in Bratislava, pp. 307-316.

Bartošová, J. \& Forbelská, M. (2010). Comparison of Regional Monetary Poverty in the Czech and Slovak Republic. Herl`any, Slovakia 13.10.2010 - 15.10.2010. In: Conference on Social Capital, Human Capital and Poverty in the Regions of Slovakia 2010. Košice: Technical University Košice, pp. s. 76-84.

Fiala, T. \& Langhamrová J. (2011). ICT Experts in the Czech Republic - Development in the Past and Future Prospects. Jindřichův Hradec 07.09.2011 - 09.09.2011. In: $19^{\text {th }}$ Interdisciplinarity Information Management Talks IDIMT-2011. Linz: Trauner Verlag universitat, pp. 51-58.

Fiala, T. \& Langhamrová J. (2010). Population Projection of the Numer and Age Structure of ICT Experts in the Czech republic. Jindřichův Hradec 08.09.2010 - 10.09.2010. In: $18^{\text {th }}$ Interdisciplinarity Information Management Talks. IDIMT-2010. Linz: Trauner Verlag universitat, pp. 115-123.

Guttman, N. B. (1993). The Use of L-moments in the Determination of Regional Precipitation Climates. Journal of Climate, Vol. 6, pp. $2309-2325$.

Hosking, J. R. M. (1990). L-moments: Analysis and Estimation of Distributions Using Linear Combinations of Order Statistics. Journal of the Royal Statistical Society (Series B), Vol. 52, No. 1, pp. 105 - 124.

Hosking, J. R. M. \& Wales, J. R. (1997). Regional Frequency Analysis: An Approach Based on L-moments. New York, Cambridge University Press.

Kyselý, J. \& Picek, J. (2007). Regional Growth Curves and Improved design Value Estimates of Extréme Precipitation Events in the Czech Republic. Climate Research, Vol. 33, pp. 243 - 255.

Löster, T., Langhamrová, J. (2011). Analysis of Long-Term Unemployment in the Czech Republic. Prague 22.09.2011 23.09.2011. In: International Days of Statistics and Economics at V̌̌E 2011. Slaný: Melandrium, pp. 228-234.

Malá, I. (2011). The use of finite mixtures of lognormal distributions in the modeling of incomes in the Czech Republic. Research Journal of Economics, Business and ICT, Vol. 4, No. 1, pp. 41-46.

Miskolczi, M., Langhamrová, J. \& Langhamrová, J. (2011). Recognition of Differentiation in Unemployment Trends Among Regions in the Czech Republic. Jindřichův Hradec 07.09.2011 - 09.09.2011. In: $19^{\text {th }}$ Interdisciplinarity Information Management Talks IDIMT-2011. Linz: Trauner Verlag universitat, pp. 387-388.

Řezanková, H., Löster, T. \& Hušek D. (2011). Evaluation of Categorical Data Clustering. Fribourg 26.01.2011 - 28.01.2011. In: $7^{\text {th }}$ Atlantic Web Intelligence Conference Advances in Intelligent Web Mastering - 3. Berlin: Springer Verlag, pp. $173-182$. 